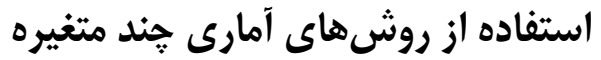

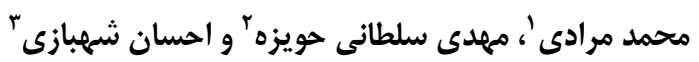

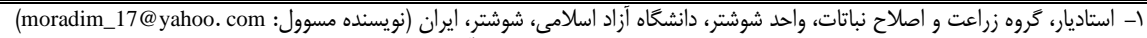

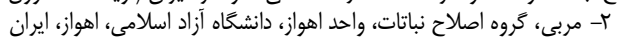

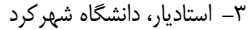

9ه///V تاريخ بذيرش

تاريخ دريافت:

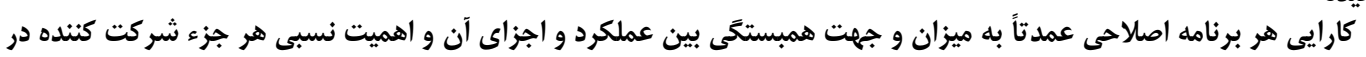

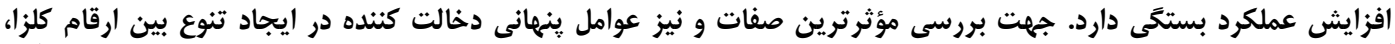

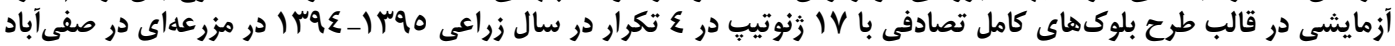

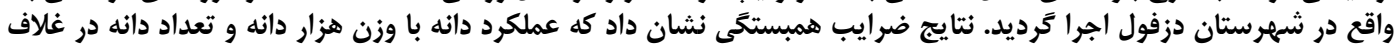

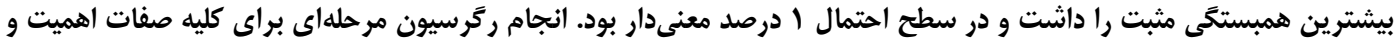

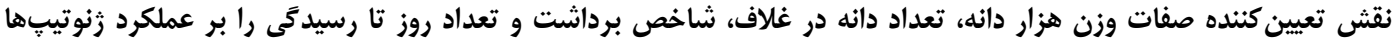

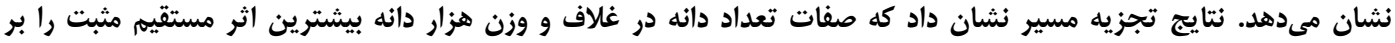

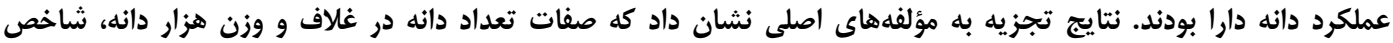

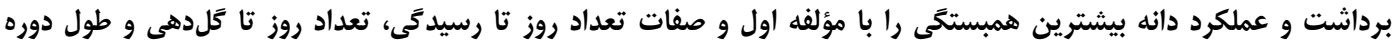

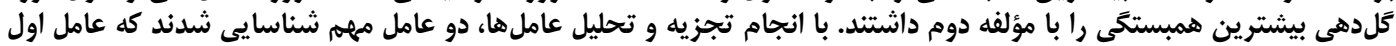

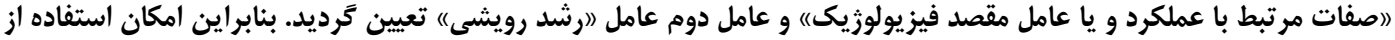

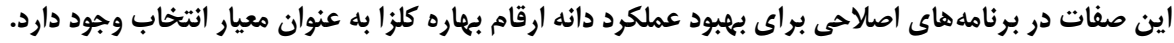

وازههاى كليدى: همبستكى، تجزيه عليت، روشهاى آمارى جند متغيره و كلزا

افزايش يا كاهش عملكرد دانه كلزا مؤثر مىباشند (ج) (ب). در

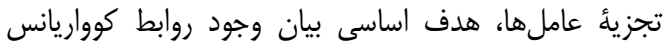

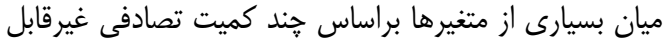

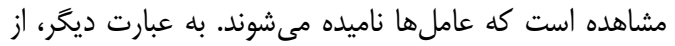

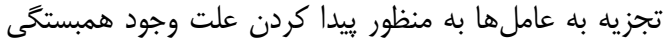

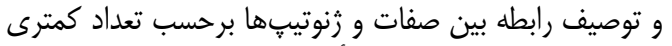

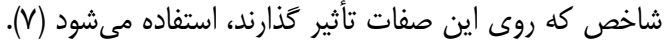

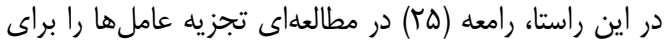

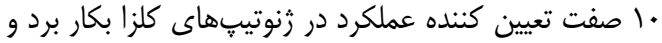

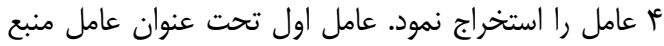

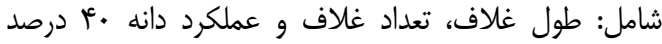

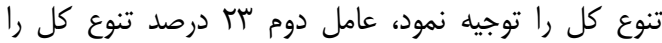

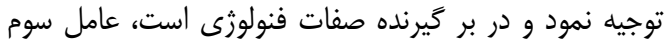

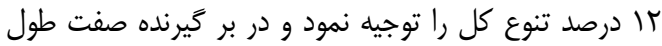

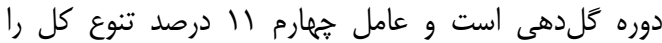

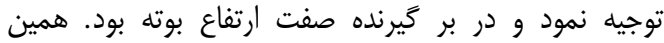

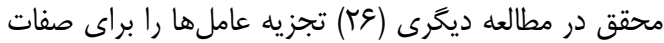

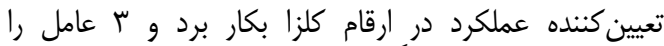

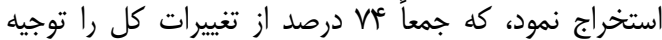

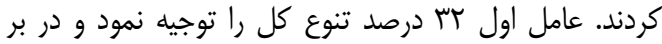

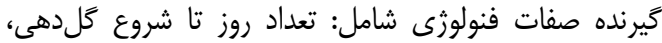

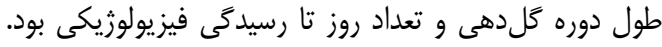

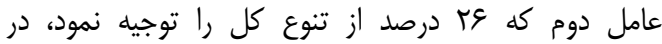

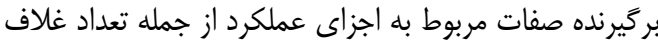

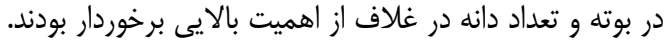

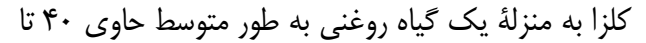

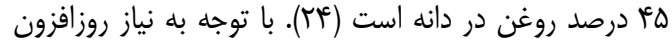

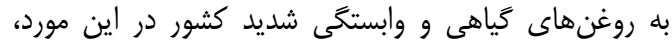

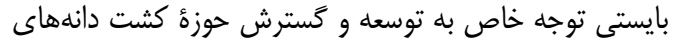

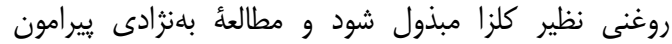

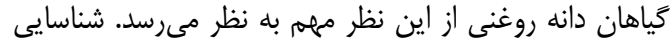

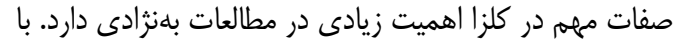

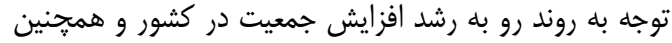
نياز روزافزون به افزايش توليد محصولات كشاورزى در در وراند

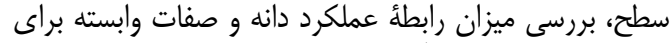

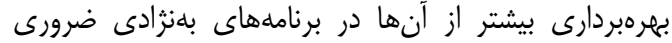

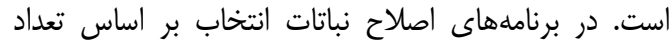

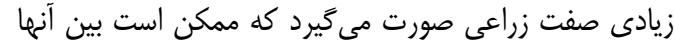

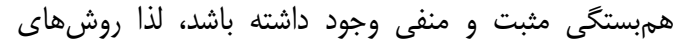

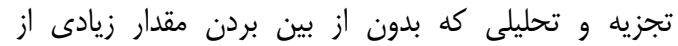

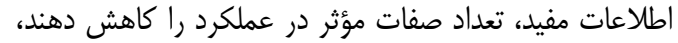

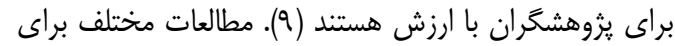

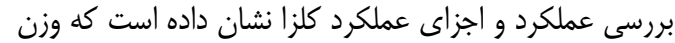

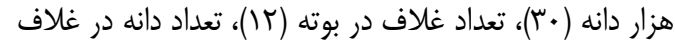

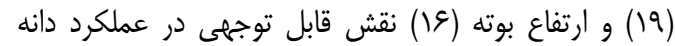

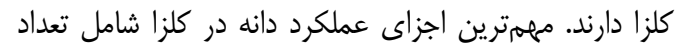

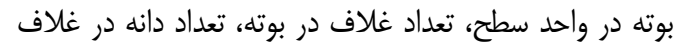

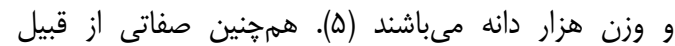
شاخص برداشت، ارتفاع بوته و تعداد غلاف دافن ساقه اصلى نيز در 


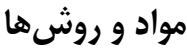

اين ثيروهش به منظور ارزيابى ارتباط صفات موردات مورد مطالعه

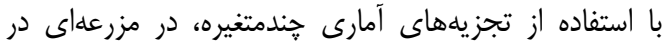

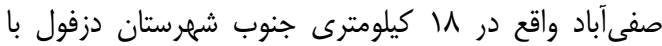

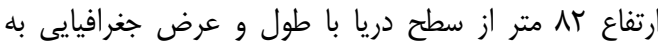

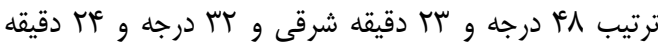
شمالى در قالب طرح بلوكهاى كامل تصادفى در هِيهار تكرار

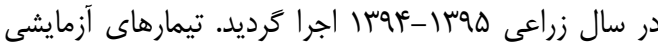

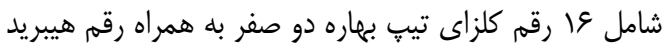

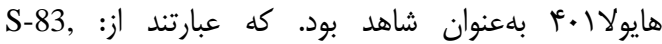
RG4403, Amica, RGAS0324, RGS006, Hyola401, Kimberley, RG405/02，RG405/03 Sarigol, Hysun110, RGS003, Hyola420, Hyola308, Hyola60, pF, Option500,

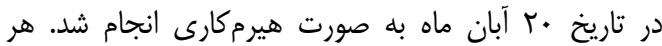

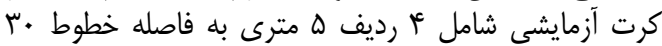

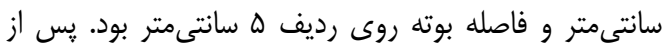

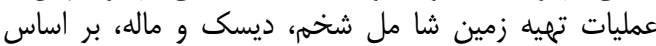

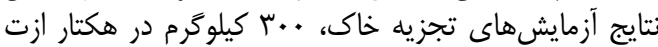

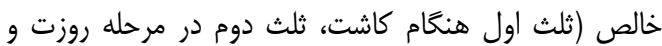

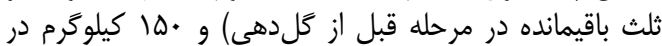

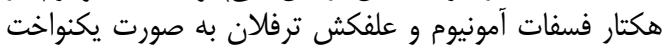

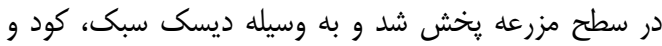

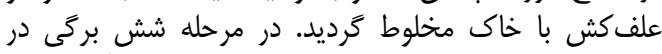

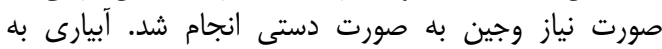

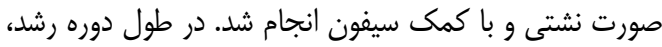

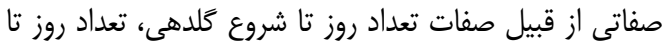

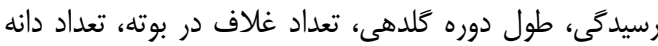

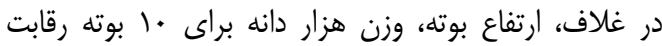

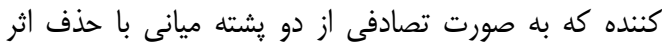

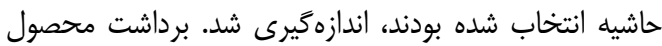

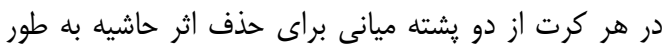

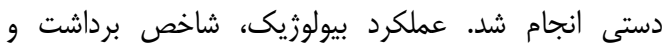

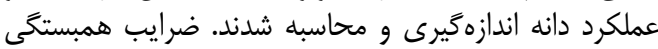

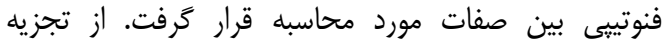

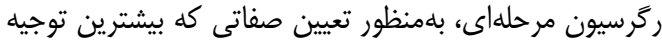

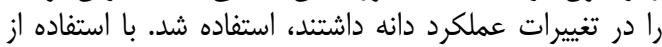

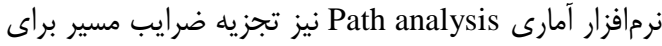

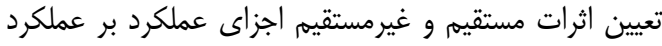

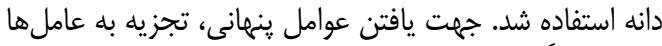

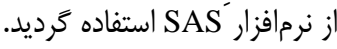

ضرايب همبستخى بينى صفات ايبى در ارقام كلزاى مورد

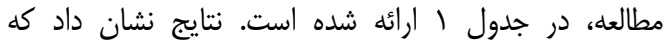

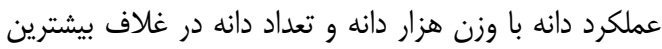

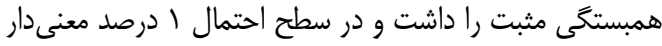

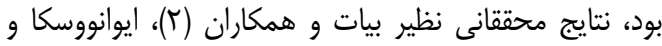

عامل سوم ها درصد تنوع كل را توجيه نمود و در بر كيرنداه

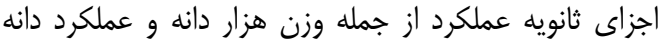

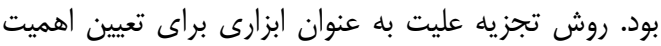

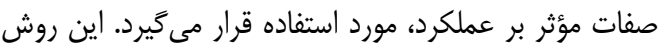

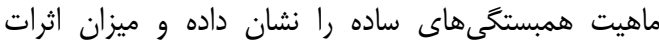

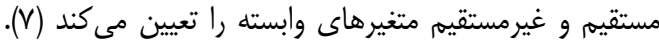

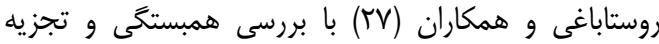

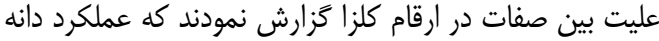

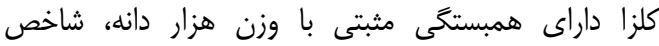

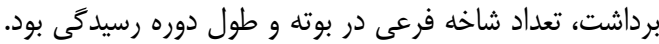

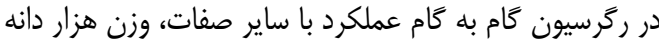

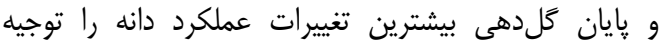

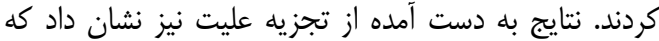

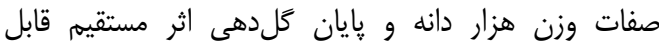

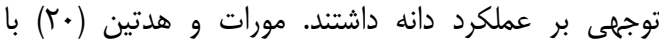

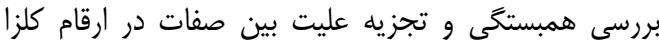

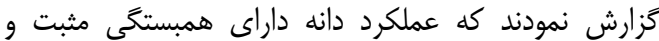
معنىدارى با صفات وزن هزار دانه، تعداد شاخه فرعى داند در بوتهاته،

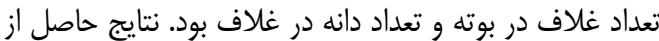

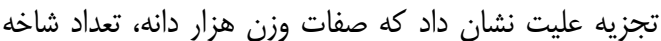
فرعى در بوته، تعداد غلاف دئ در درئ بوته

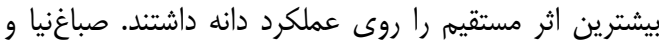

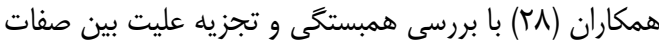

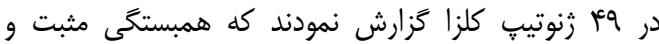

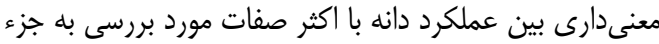

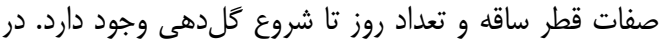

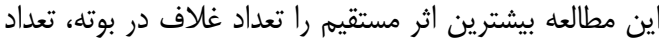

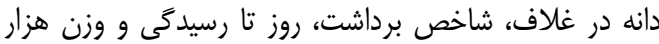

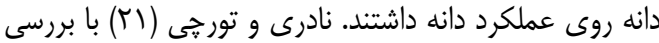

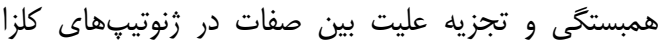

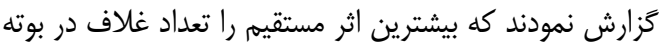

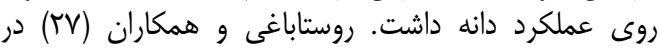

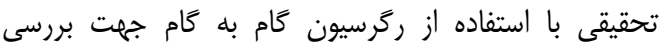

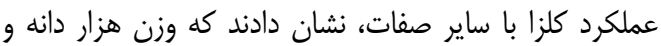

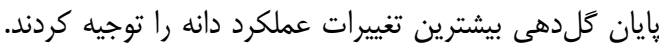

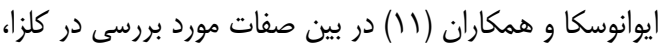

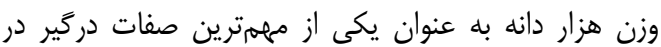

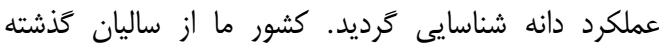

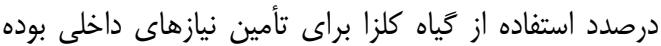

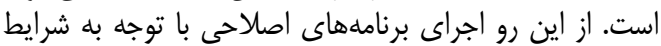

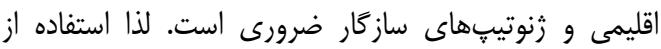

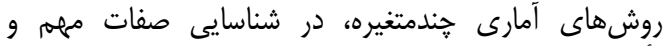

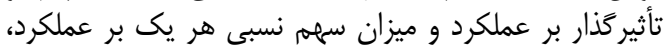

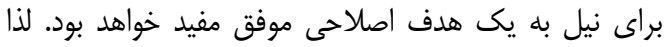

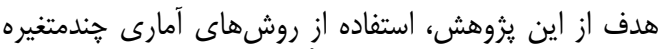

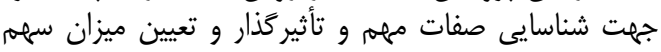

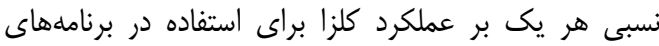

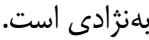


زودتر و به موقع، آغاز يا تكميل شدهاند و به عبارتى إز إنى نظر

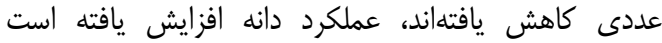

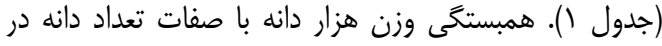

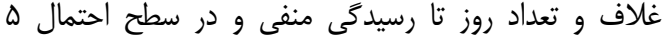

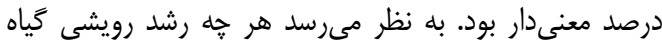

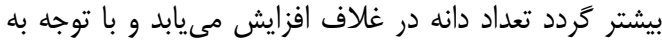

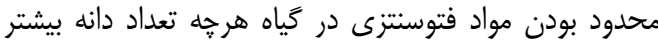

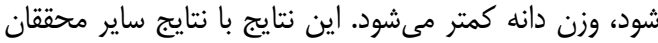

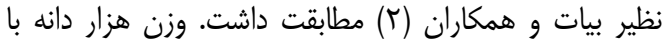

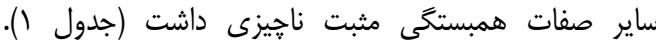

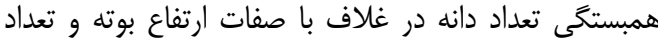

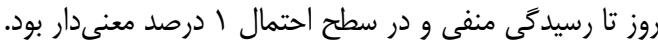

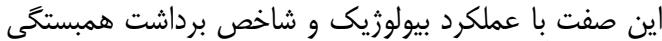

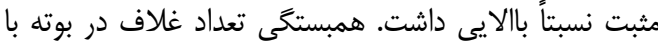

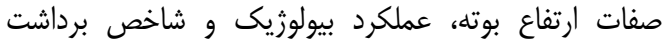

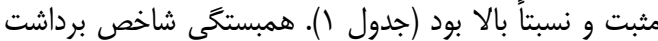

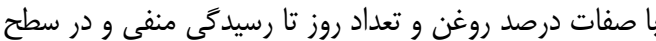

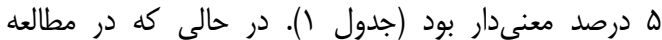

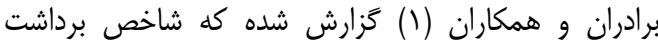

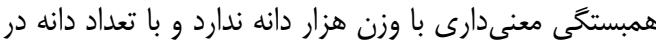

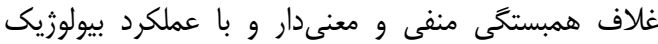
همبستخى مثبت و معنى دارى دارد. ايوانوسكا و همكاران (11)

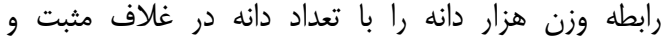

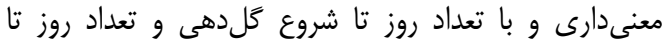
خاتمه كلدهى منفى و معنى دار عنوان كردند. تعاع.

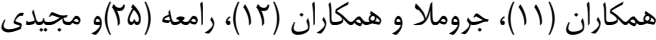

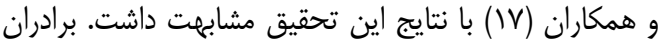

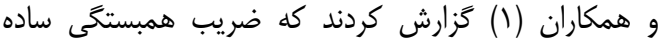

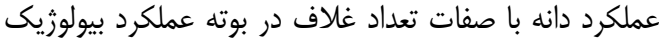

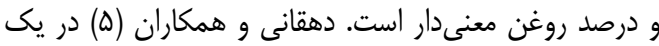

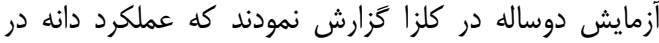

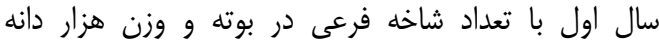

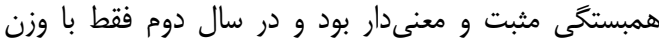

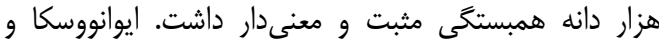

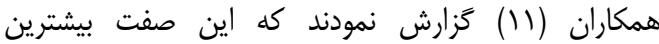

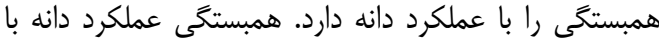

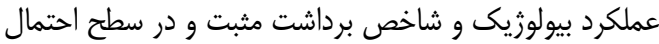

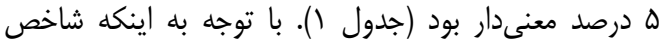

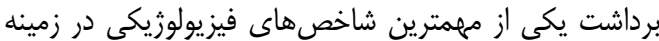

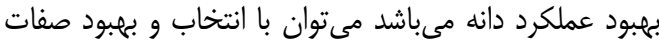

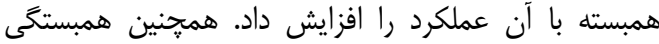

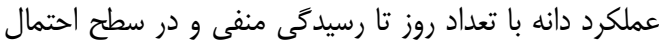

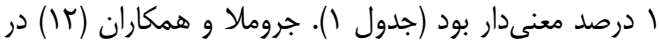

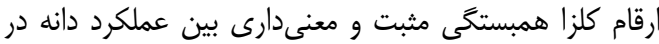

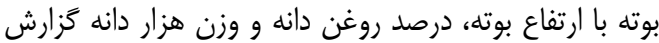

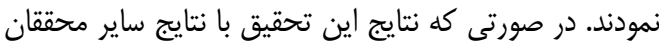

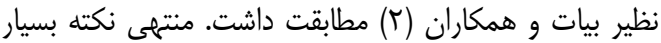

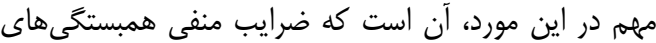

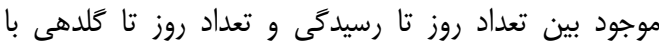

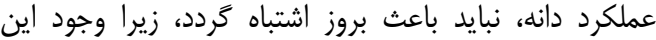

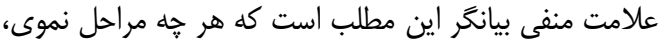

جدول ا- ضرايب همبستخى بين صفات مورد بررسى در IV رقم بهاره كلزا Table 1. Correlation coefficients among the studied traits in 17 spring canola cultivars

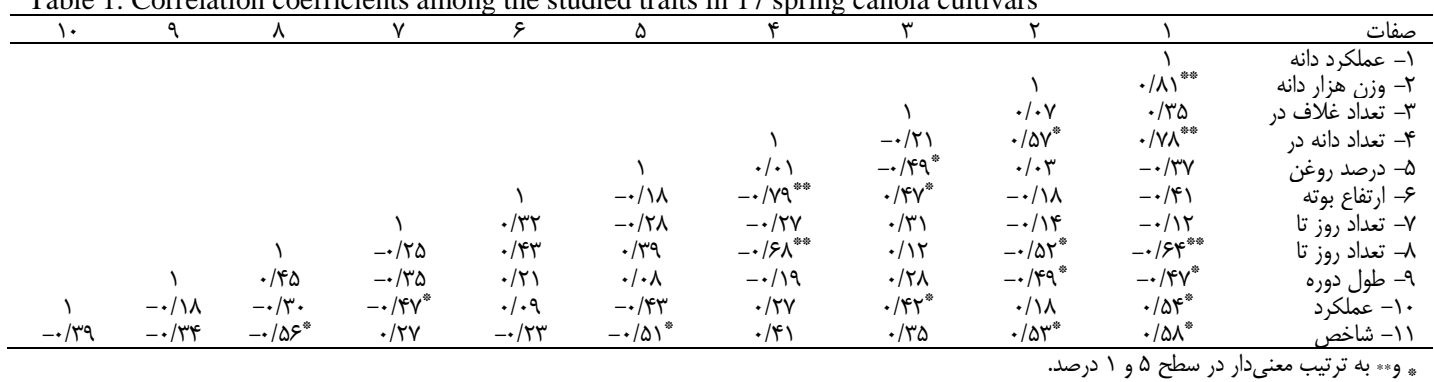

با توجه به نتايج حاصل از رگرسيون مرحلهاى براى

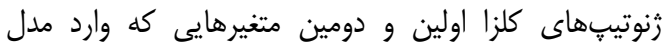

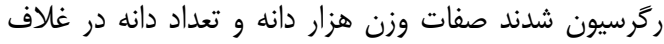

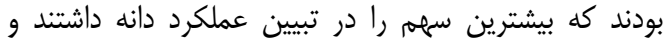

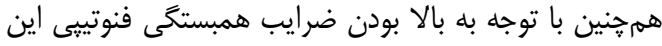

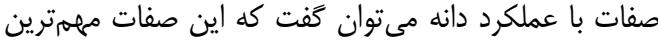

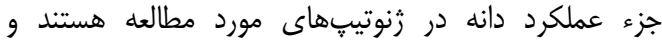

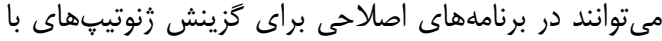
عملكرد بالاتر مورد توجه قرار گيردا

\section{تجزيه رَّرسيون مرحلهاى}

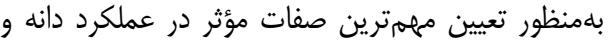

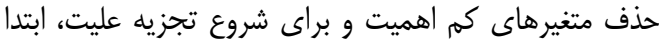

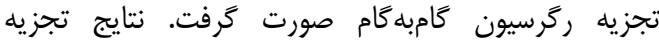

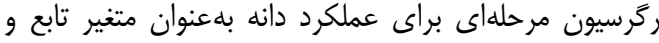

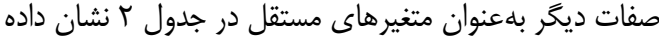

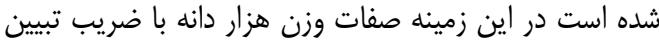

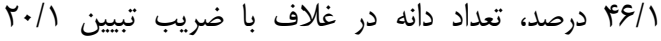

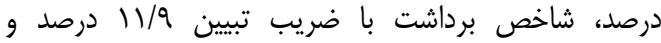

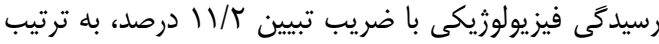

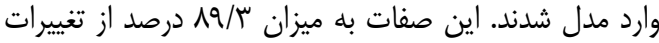

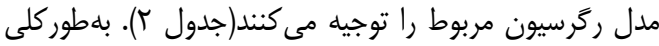


جدول r- نتايج رگرسيون مرحلهاى براى عملكرد دانه به عنوان متغير وابسته و ساير صفات به عنوان متغير مستقل Table 2. Results of stepwise regression for grain yield as dependent variable and other traits as independent variable

\begin{tabular}{|c|c|c|c|c|c|}
\hline $\mathrm{R}^{r}$ & $\mathrm{~F}$ & خطاى استاندارد & ضريب ركر سيون جزء & متغير وارد شده & مرحله ورود متغير \\
\hline.$/ 481$ & $1 F T / 10^{*}$ & $F T / \backslash 9$ & $\cdot / 481$ & وزن هزار دانه & 1 \\
\hline | /99T & $q \mu / V .^{* * *}$ & $r V / \Delta)$ & $\cdot / 4 \cdot 1$ & تعداد دانه در غلاف & r \\
\hline$\cdot /$ VAI & $r \Delta / / V^{*}$ & 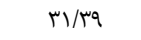 &.$/ 119$ & شاخص برداشت & r \\
\hline ./194 & $19 / \cdot 0^{*}$ & 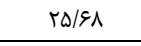 &.$/ 114$ & رسيدگى فيزيولوزيكى & i \\
\hline
\end{tabular}

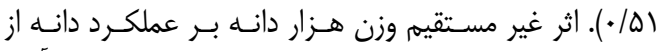

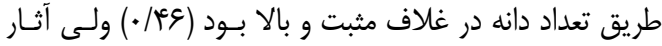

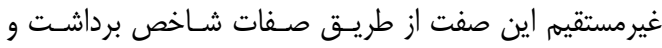

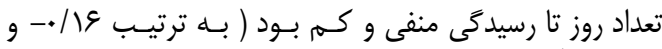

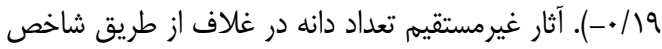

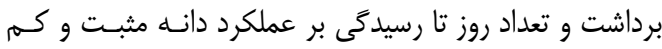

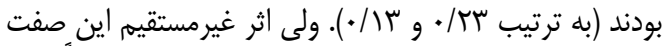

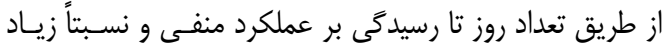

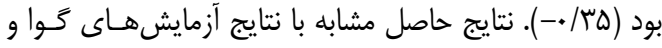

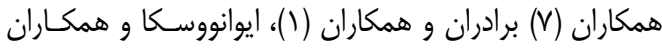

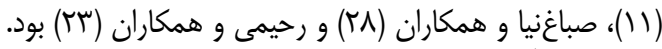

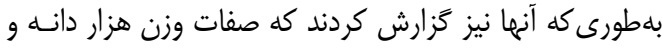

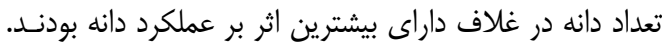

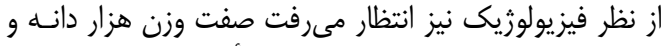

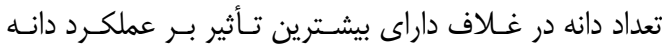

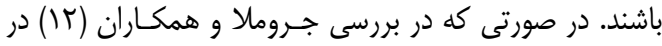

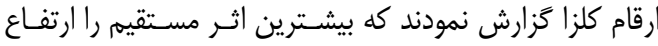

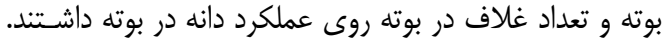

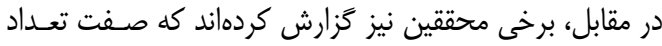

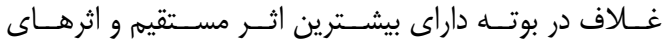

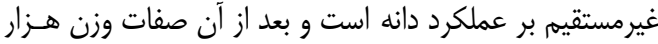

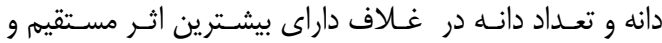

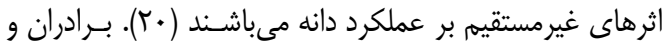

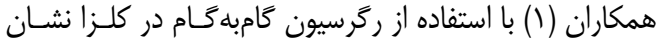

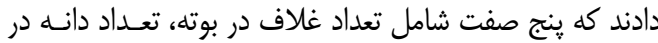

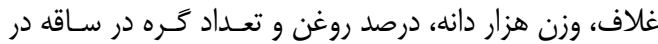

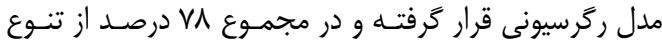

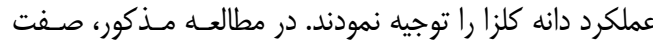

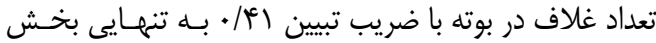

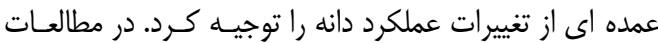

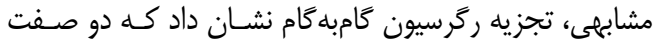

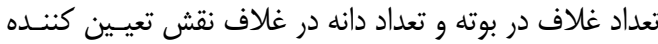

و مهمى در توجيه عملكرد دانه دارند (1).
بنابراين مىتوان استنباط نمود كه صفات وزن هزار دانه و

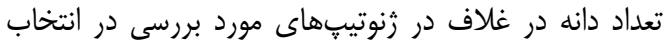

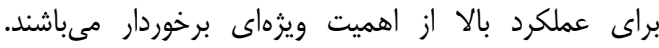

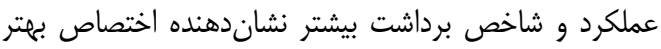

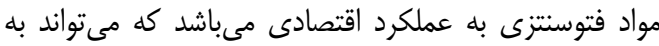

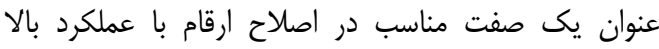

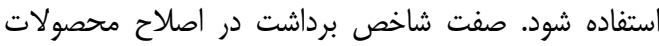

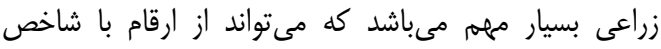

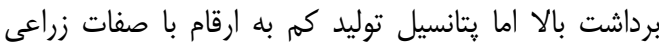

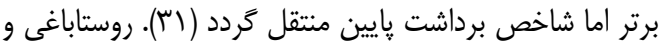

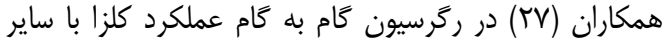

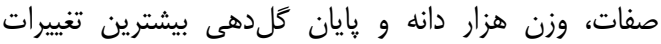

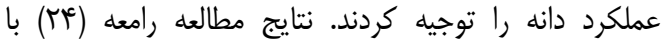

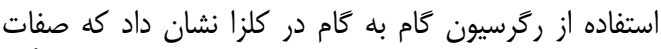

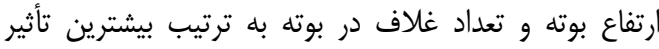

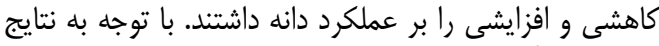

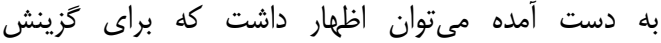

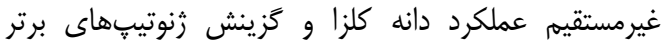

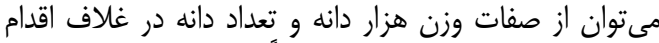

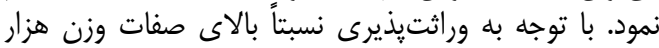

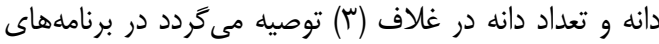

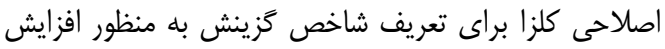
تمملكرد دانه از اين صفات استفادي تعريف شراخد تجزيه عليت

بلمنظور درك بهتر و تفسير دقيقتر نتايج به دست آمده از

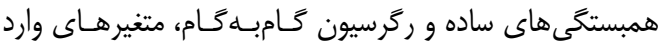

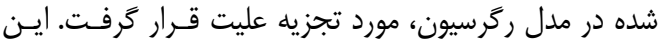

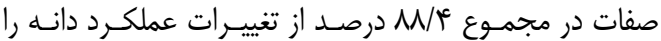

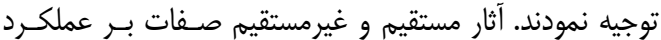

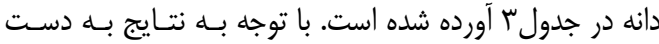

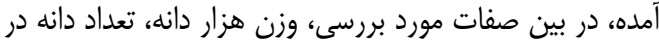

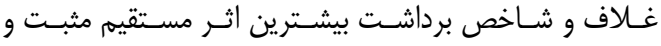

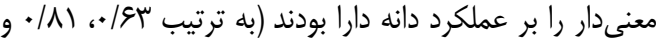


جدول سـ آثار مستقيم (روى قطر) و غيرمستقيم وزن هزار دانه، تعداد دانه در غلاف، در صد روغن و ارتفاع بوته بر عملكرد دانه Table 3. Direct (on the diagonal) and indirect effects of 1000-seed weight, number of seeds per pod, oil percent and plant height on grain yield

\begin{tabular}{|c|c|c|c|c|c|c|}
\hline اثرات باقيمانده & ضريب همبستكى بانه & تعداد روز تا & شراشت & تعدراد دانه & وزن هزار دانه & \\
\hline & $\cdot|\Lambda|^{-m}$ &.$- / 19$ & -.019 & .148 & $\cdot \mid q^{\mu}$ & وزن هزار دانه \\
\hline &.$/ \mathrm{V} \wedge^{* m}$ &.$- / \Gamma \Delta$ & 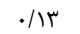 & $\cdot|\Lambda|^{*}$ &.$/ \pi r$ & تعداد دانه در غلاف \\
\hline & $\cdot \mid \Delta \Lambda^{*}$ &.$/ 1$. & $\cdot|Q|^{\prime \prime \prime}$ & $\overline{-\cdot \cdot \cdot \Delta}$ & r & شاخص برداشت \\
\hline 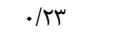 & $-.194^{* * 0}$ &.$- / \mu \Lambda^{*}$ &.$/ 11$ & . &.$- / 18$ & تعداد روز تا رسيدگى \\
\hline
\end{tabular}

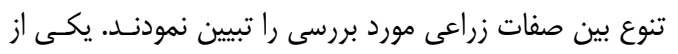

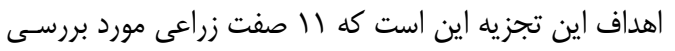

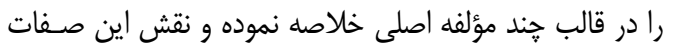

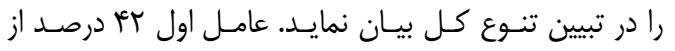

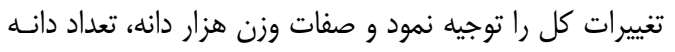

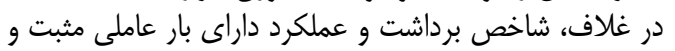

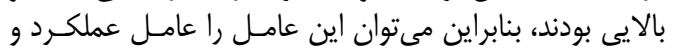

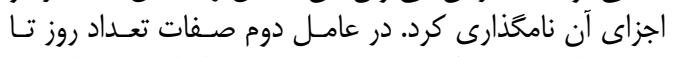

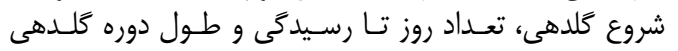

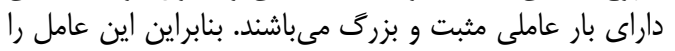

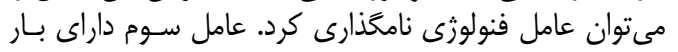

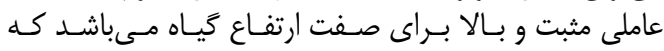

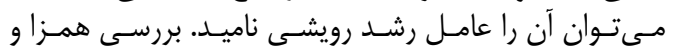

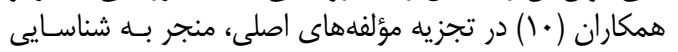

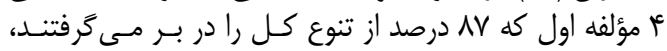
كرديد.

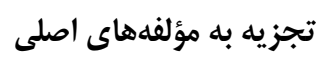

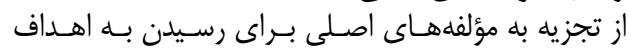

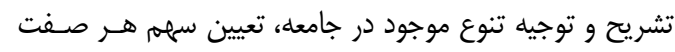

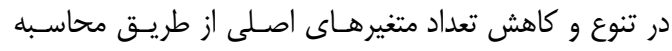

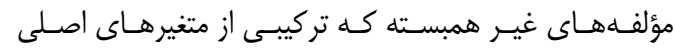

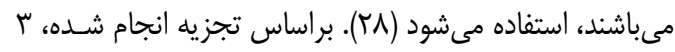

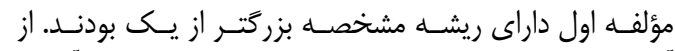

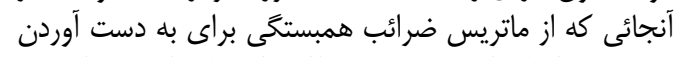

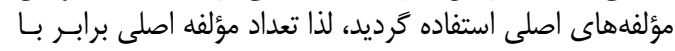

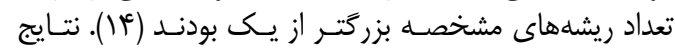

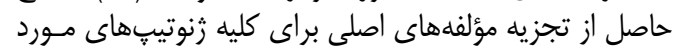

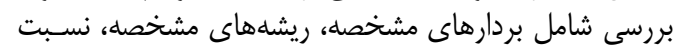

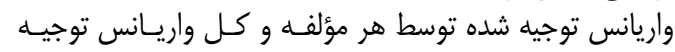

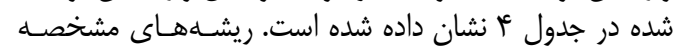

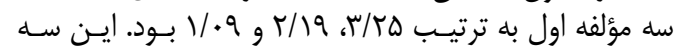

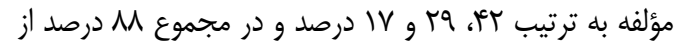

Table 4. Eigen vectors, Eigen values, Portion and the cumulative variance percent

جدول عا- بردارهاى مشخصه، ريشهاى مشخصه، نسبت واريانس توجيه شده توسط هر مؤلفه و نسبت تجمعى

\begin{tabular}{|c|c|c|c|}
\hline \multirow[b]{2}{*}{ مؤ مؤلفه سوم } & \multicolumn{2}{|c|}{ بردارهاى مشخصه } & \multirow{2}{*}{ صفات } \\
\hline & مؤلف دوم & مؤلفه اول & \\
\hline זr & .1 .9 & $\cdot / \Lambda \Delta$ & وزن هزار دانه \\
\hline .119 &.$/ r$ & .199 & تعداد دانه در غلاف \\
\hline.$- / 11$ & - /NA & $-\cdot / \cdot V$ & تعداد روز تا رسيدگى \\
\hline $.1 \cdot \mathrm{V}$ &.$- / 10$ &.$/ \mathrm{rr}$ & شاخص برداشت \\
\hline .1 .0 & ./ve & $-(r)$ & طول دوره كلدهى \\
\hline.$/ 1 F$ & $\cdot 1 \cdot 1$ & E & تعداد غلاف در بوته \\
\hline.$/ 1 \Delta$ & $\cdot / \mathrm{V} q$ & -.1 .9 & تعداد روز تا شروع كَلدهى \\
\hline .1 .9 &.$/ T A$ &.$/ M$ & عملكرد بيولوزيك \\
\hline-.1 .9 &.- .1 .9 & rות & درصد روغن \\
\hline$\cdot|\Lambda|$ & . $/ \mathrm{r}$ & $-\pi$ & ارتفاع بوته \\
\hline.$/ \cdot \mathrm{V}$ & .1 .4 & $\cdot / \Delta \&$ & عملكرد دانه \\
\hline.$/ \mathrm{V}$ &.$/ 49$ & . LT & نسبت واريانس توجيه شده \\
\hline$\cdot / \mathrm{M}$ & $\cdot|r|$ & . LT & واريانس توجيه شده تجمعى \\
\hline $1 / .9$ & $5 / 19$ & $T / T \Delta$ & ريشه مشخصه \\
\hline
\end{tabular}

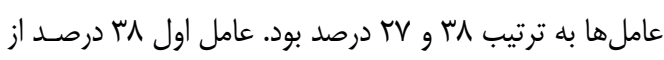

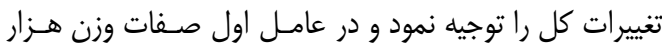

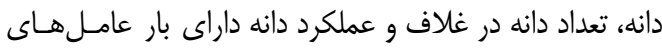

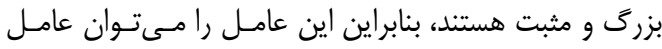

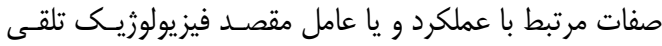

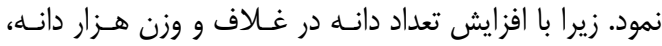

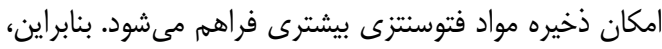

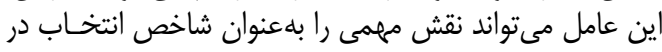

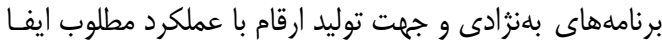

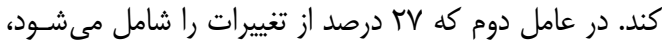

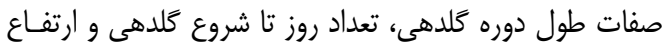

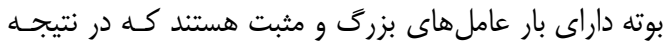

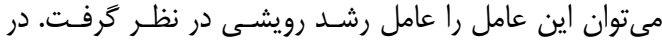

تجزيه عاملها

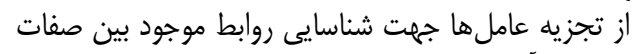

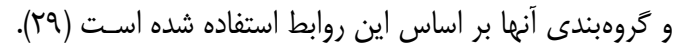

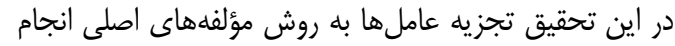

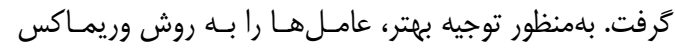

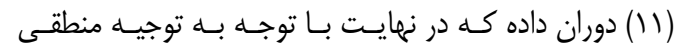

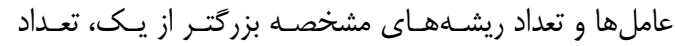

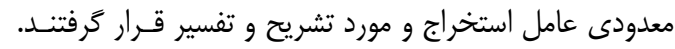

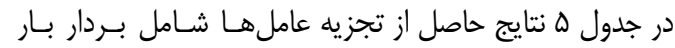

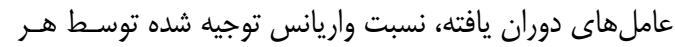

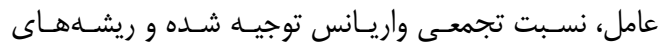

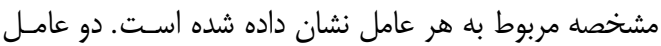

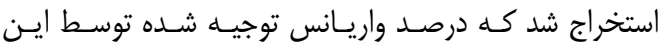




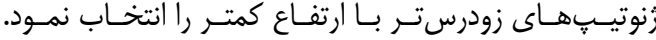

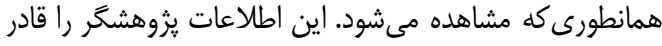

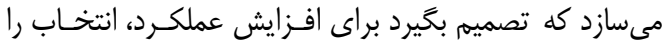

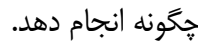

واقع اين متغيرهاى فنولوزيك با اثر روى صفات، رشد رويشى

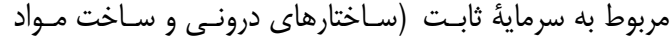

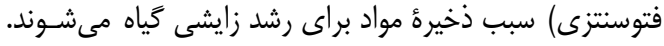

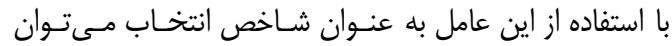

جدول هـ- بردار بار عاملهاى دوران يافته، نسبت واريانس توجيه شده توسط هر عامل، نسبت تجمعى واريانس توجيه شده و ريشهاى مشخصه Table 5. Rotated factor loadings, the proportion of variation contributed by each factor, Cumulative percentage of variance and Eigen-values

\begin{tabular}{|c|c|c|}
\hline \multicolumn{2}{|c|}{ بار عامل هاى } & صفات \\
\hline$\frac{203}{-.1 \cdot 0}$ & $\frac{1.1}{.19 \mathrm{~V}}$ & وزن هزار دانه \\
\hline .1 .9 & $\cdot / V T$ & تعداد دانه در غلاف \\
\hline.$|9|$ &.$- / \cdot \Delta$ & تعداد روز تا رسيدگى \\
\hline.$/ 14$ &.$/ 1 r$ & شاخص برداشت \\
\hline$\cdot / V^{\mu}$ &.$- / 19$ & طول دوره كلدهى \\
\hline .1 .9 &.$/ \Lambda$ & تعداد غلاف در بوته \\
\hline$\cdot \mid \& \Delta$ &.$- / 49$ & تعداد روز تا شروع كلدهى \\
\hline 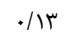 & r & عملكرد بيولوزيك \\
\hline 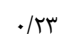 & $\cdot / 1 \wedge$ & درصد روغن \\
\hline$\cdot / V 1$ &.$/ 19$ & ارتفاع بوته \\
\hline.$/ 10$ & .190 & عملكرد دانه \\
\hline rr & « & نسبت واريانس توجيه شده \\
\hline 90 & ی & واريانس توجيه شده تجمعى \\
\hline $1 / \mathrm{rA}$ & $r / g r$ & ريشه مشخصه \\
\hline
\end{tabular}

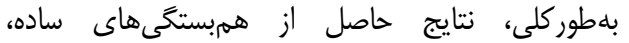

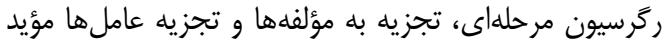

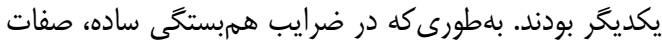

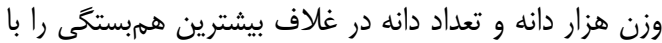

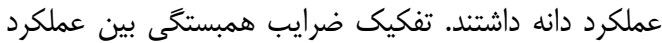

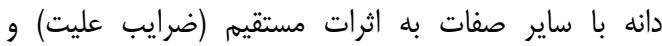

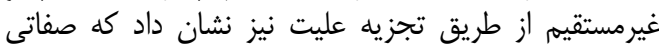

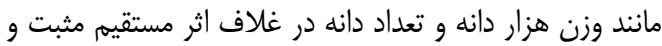

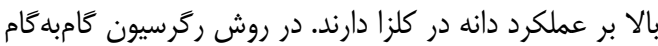

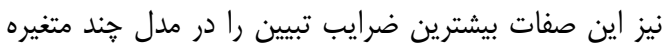

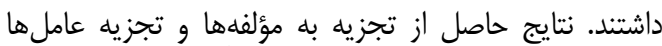

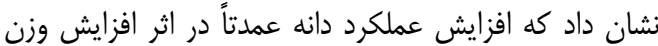

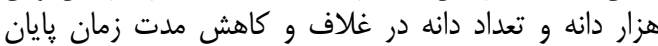

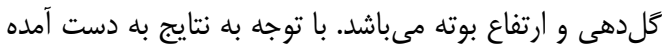

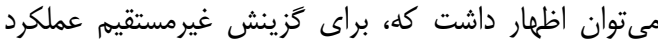

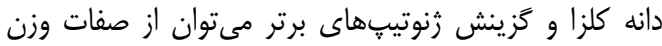

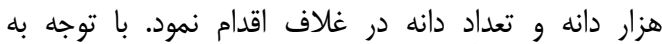

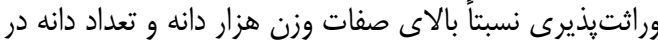

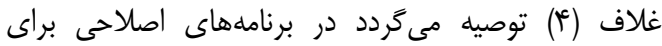

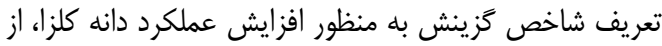
اين صفات استفاده كردد.

هزينههاى مربوط به اجراى اين تحقيق از محل إنى اعتبارات

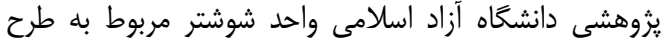

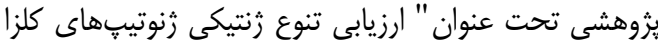
(Brassica napus L)

جند متغيره" تأمين شده است كه بدينوسيله تشكر مى تردئ
استفاده از روش خُندمتغيره تجزيه به عامل ها در شناسـايى

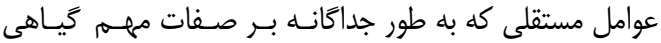

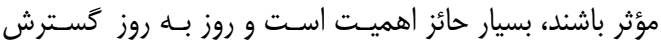

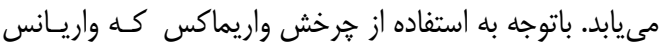

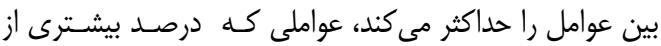

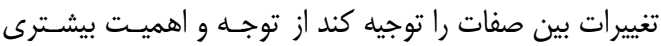

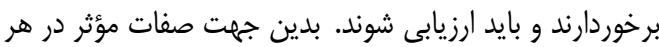

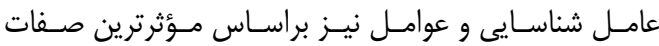

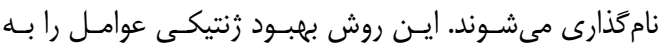

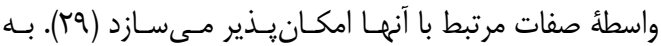

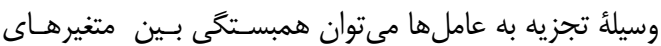

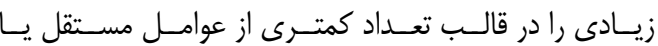

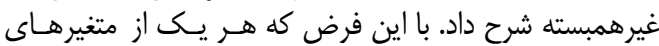

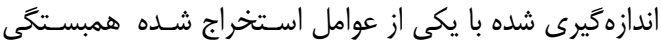

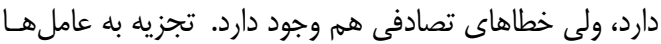

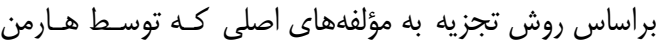

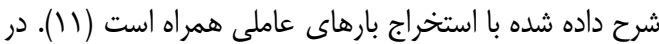

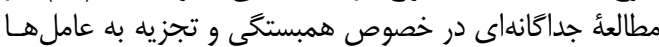

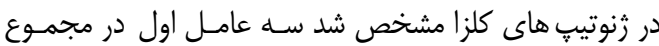

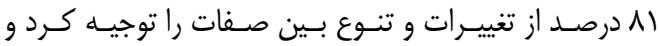

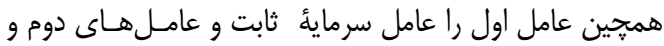

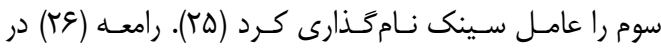

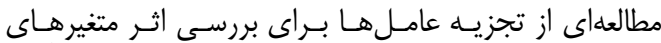

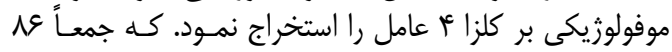

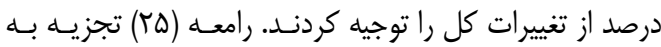

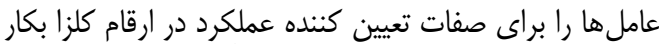

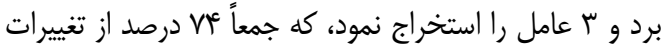
كل را توجيه كردند. 
1. Baradaran, R.,E Majidi, F. Darvish and M. Azizi. 2006. Study of correlation relationships and pe coefficient analysis between yield and yield components in rapeseed (Brassica napus L.). Journal of Agricultural Sciences, 12: 811-819.

2. Bayat, M., B. Rabiei, M. Rabiee and A. Moumeni. 2008. Assessment of relationship between grain yield and important agronomic traits of rapeseed as second culture in paddy fields. Journal of Crop Production and Processing, 12: 475-486 (In Persian).

3. Brandle, J.K. and P.B.E. Mcvetty. 1989. Heterosis and combining ability in hybrids derived from oilseed rape cultivars and inbred lines. Crop Science, 29: 1191-1195.

4. Clark, J.M. and G.M. Simpson. 1978. Growth analysis of Brassica napus. Canadian Journal of Plant Science, 58: 587-597.

5. Dehghani, H., H. Omidi and N. Sabaghnia. 2008. Graphic analysis of relation of rapeseed using the biplot method. Agronomy Journal, 100: 1443-1449.

6. Farshadfar, A. 1996. Principle and multivariate methods. Razi University press. Kermanshah, 382 pp (In Persian).

7. Guo, J.C., X.X. Guo and R.H. Liu. 1987. A study of correlations between yield components in mutants of Brassica napus L. Oil Crops of China, 2: 23-25.

8. Guertin, W.H. and J.P. Bailey. 1982. Introduction to Modern Factor Analysis. Edwards Brothers Inc, $265 \mathrm{pp}$.

9. Hamza, S., W.B. Hamida, A. Rebai and M. Harrabi. 2004. SSR-based genetic diversity assessment among Tunisian winter barley and relationship with morphological traits. Euphytica, 135: 107-118.

10. Harman, H.H. 1976. Modern Factor Analysis, Third Edition Revised. University of Chicago Press, $495 \mathrm{pp}$.

11. Ivanovska, S., C. Stojkovski, Z. Dimov, A. Marjanovic-Jeromela, M. Jankulovska and L. Jankuloski. 2007. Interrelationship between yield and yield related traits of spring canola (Brassica napus L.) genotypes. Genetika, 39(3): 325-332.

12. Jeromela, A.M., R. Marinkovic., A. Mijic., Z. Zdunic and M. Jankulovska. 2008. Correlation and path analysis of quantitative traits in winter rapeseed (Brassica napus L.). Agric. Conspec. Sciense, 73: 1318.

13. Johnson, R.A. and D.W. Wichern. 1992. Applied multivariate statistical analysis. 3rded. Englewood Cliffs, NJ: Prentice Hall, $767 \mathrm{pp}$

14. Kakaei, M., A. Zebarjadi, A. Mostafaie and A. Rezaeizad. 2014. Genetic variation and traits interrelationship in some rapeseed genotypes using multivariate techniques under two moisture conditions. Journal of Applied Crop Breeding, 2: 31-45 (In Persian).

15. Khan, F.A., S. Ali, A. Shakeel, A. Saeed and G. Abbas. 2006. Correlation analysis of some quantitative characters in Brassica napus L. Journal of Agricultural Research, 44: 7-14.

16. Leilah, A.A. and S.A. Al-Khateeb. 2005. Yield analysis of canola (Brassica napus L.) using some statistical procedures. Saudi Journal of Biological Sciences, 12: 103-112.

17. Majidi, M.M., M. Jafarzadeh Ghahdarijani, F. Rashidi and A. Mirlohi. 2016. Relationship of different traits in rapeseed (Brassica napus L.) cultivars under normal and drought conditions. Journal of Crop Breeding, 8: 65-55 (In Persian).

18. Manly, B.F.J. 2004. Multivariate Statistical Methods a Primer. 3rd ed., Chapman \& Hall/CRC Inc., $226 \mathrm{p}$.

19. Mohammadi, S.A. and B.M. Prasanna. 2003. Analysis of genetic diversity in crop plants salient statistical tools and considerations. Crop Science, 43: 1235-1248.

20. Murat, T. and C. Vahdettin. 2007. Relationships between yield and some yield components in rapeseed (Brassica napus L.) cultivars by using correlation and path analysis. Pakistan Journal of Botany, 39: 81-84.

21. Naderi, R. and M. Toorchi. 2012. Path analysis of the relationships between yield and some related traits in canola (Brassica napus L.) under salinity stress conditions. Annals of Biological Research, 3: $1731-1734$

22. Naseri, F. 1990. Oil seed crops. Astan-ghods razavi press. Mashhad, 823 pp (In Persian).

23. Rahimi, M., Ramezani, M. and Ozoni Davaji, A. 2016. Investigation of path and correlation analysis of pattern and plant densities effect on two rapeseed cultivars. Journal of Crop Breeding, 8(19): 218227. (In Persian).

24. Ramee, V. 2012. Correlation and factor analyses of quantitative traits in rapeseed (Brassica napus L.). Agriculture Innovations and Research, 1: 2319-1473.

25. Rameeh, V. 2013. Multivariate analysis of some important quantitative traits in rapeseed (Brassica napus L.) advanced lines. Journal of Oilseed Brassica, 4: 75-82.

26. Rameeh, V. 2014. Multivariate Regression Analyses of Yield Associated Traits in Rapeseed (Brassica napus L.) Genotypes. Advances in Agriculture Article ID, 6: 264-345.

27. Roostabaghi, B., H. Dehghan, B. Alizadeh and N. Sabaghnia. 2013. Study of diversity and evaluation of relationships between yield and yield components of rapeseed via multivariate methods. Journal of Crop production and processing, 2: 53-63 (In Persian).

28. Sabaghnia, N., H. Dehghani, B. Alizadeh and M. Moghaddam. 2010. Interrelationships between seed yield and 20 related traits of 49 canola (Brassica napus L.) genotypes in non-stressed and waterstressed environments. Spanish Journal of Agricultural Research, 8: 356-370.

29. Seiler, G.J. and R.E. Stafford. 1979. Factor analysis of components of yields in guar. Crop Science, 25: $905-908$.

30. Sharma, S. 1996. Applied multivariate techniques. 1nd ed. John Wiley and Sons, 493 pp.

31. Vafaei, S.N., A. Tobeh, A. Taee and S. Jamaati-e-Somarin. 2010. Study of phenology, harvest index, yield, yield components and oil content of different cultivars of rain-fed safflower. World Applied Science Journal, 8: 820-827. 


\title{
Study the Relations between Grain Yield and Related Traits in Canola by Multivariate Analysis
}

\author{
Mohammad Moradi ${ }^{1}$, Mehdi Soltani Hoveize ${ }^{2}$ and Ehsan Shahbazi ${ }^{3}$ \\ 1- Assistant Professor, Department of Agronomy and Plant Breeding, Shoushtar Branch, Islamic Azad University, \\ Shoushtar, Iran (Corresponding Author: moradim_17@yahoo.com) \\ 2- Instructore, Department of Plant Breeding, Ahvaz Branch, Islamic Azad University, Ahvaz, Iran \\ 3- Assistant Professor, College of Agriculture, Shahrekord University \\ Received: June 17, 2016 \\ Accepted: August 28, 2016
}

\begin{abstract}
The efficiency of a breeding program depends mainly on the direction of the correlation between yield and its components and the relative importance of each component involved in contributing to seed yield. The objective of this study was to detection of traits affecting canola yield by multivariate analysis, at Khozestan Province, Iran, in the agricultural year in 20152016. A randomized complete block design with four replications was used. The results of stepwise regression analysis revealed that 1000-grain weight, number of pods per plant, HI and days to maturity significantly had more important effects respectively on seed yield. The results of path analysis indicated that the number of grain per pod and 1000-grain weight had the largest direct effects on the grain yield. According to the results of the principal component analysis, PC1 was moderately correlated with number of seeds per pod, 1000-seed weight, HI and seed yield. PC2 was moderately correlated with days to flowering, days to maturity and flowering period. The results of factor analysis exhibited two factors including sink factor (number of seeds per pod, 1000-seed weight and seed yield) and fixed capital factor (phonological traits). It seems that its seams possible to use these traits as selection criteria in breeding programs for improve seed yield of spring rapeseed cultivars.
\end{abstract}

Keywords: Canola, Factor Analysis, Principal Comp onents, Path analysis, Step-wais Regression 